\title{
Editorial
}

\section{Transcranial electric stimulation (tES) and Neurolmaging: the state-of-the-art, new insights and future prospects in basic and clinical neuroscience.}

\begin{abstract}
Partly due to novel and refined neuroimaging methods, transcranial electric stimulation (tES) of the brain has attracted an increased interest in the scientific community, which is well documented in an exponential growth of research articles over the last years. Being an appealing tool to manipulate brain electric or magnetic activity to study its functional role, tES also promises to allow for purposeful modulation of brain function and behavior, with all associated implications for evidence-based use of this technique in a clinical context.

However, despite remarkable research efforts, the underlying mechanisms of tES effects are still not well understood. This Special Issue aims at providing a comprehensive and up-todate overview of the state-of-the-art in combining $\mathrm{tES}$ and neuroimaging, while introducing most recent insights and outlining future prospects related to this new and rapidly growing field.

The important findings reported in this Special Issue document very impressive methodological advancements in the context of combined tES and neuroimaging studies. At the same time, they also point to the many caveats and specific challenges associated with such studies, e.g. related to artifact cancellation or anatomical variability. Besides promising to advance basic neuroscience, combined tES and neuroimaging studies may also substantially change previous conceptions about how electric or magnetic stimulation acts on the brain.
\end{abstract}

It was once said that there are no second acts in American lives (Fitzgerald, 1941). Yet perhaps due to the invention of Transcranial Magnetic Stimulation (TMS) in the 1980s - the use of direct current stimulation to investigate brain function has recently undergone something of a renaissance (Liew et al. 2014). The technical simplicity, portability and ease of application has meant that trasnscranial Electrical Stimulation (tES), also termed transcranial current stimulation (TCS), promises to be an powerful tool to explore the links between brain function and disease in both basic and clinical neuroscience.

However, despite remarkable research efforts, the underlying neurobiological mechanisms of tES' effects are still incompletely understood. In a manner similar to earlier studies combining neuroimaging and stimulation to investigate the neuronal underpinnings of TMS (Paus, 1999), a major limitation in investigating the immediate effects of tES on brain activity is the strong stimulation artifacts that accompany the application of the technique, exceeding the actual neurobiological activity by some magnitudes. In particular, the separation of the stimulator signal from unrelated physiological activity remains a great challenge.

This Special Issue aims at providing a comprehensive and up-to-date overview of the stateof-the-art in combining tES and neuroimaging. As combining stimulation and imaging is a fast evolving field, we felt that it was as important to introduce recent insights and future prospects as it was to provide a overview of current work. The issue begins with a review 
from Bergmann et al., who provide a conceptual framework to assist in interpreting the subsequent papers, emphasizing principal strategies and highlighting promising future directions to exploit the benefits of combining tES with neuroimaging or electrophysiology.

In a concurrent transcranial Direct Current Stimulation (tDCS)-Magnetoencephalography (MEG) study, Claire Hanley and colleagues investigated the effects of tDCS on both evoked and induced indices of brain activity, employing both multiple experimental tasks and electrode montages. As with a number of studies in this special issue, the authors demonstrated the feasibility of tDCS-MEG to investigate stimulation effects on brain responses, and presented evidence for the modulation of both visual and motor activity subsequent to stimulation.

In another concurrent tDCS-MEG study, Eliana Garcia Cossio and colleagues assessed the impact of tDCS on slow cortical magnetic fields (SCF) related to a classical S1-S2 paradigm. They found that anodal and cathodal tDCS had a polarity-dependent impact on reaction times and strength of SCF in primary sensorimotor and medial centro-parietal cortices. By showing for the first time that tDCS has an immediate impact on SCF of task-related areas that are identical with brain regions previously described in metabolic neuroimaging studies, they exemplify that combing tDCS and whole-head MEG is a powerful approach to investigate the direct effects of $\mathrm{tES}$ on ongoing neuromagnetic source activity, brain function and behavior.

These two studies are complemented by the paper from Tom Marshall and colleagues, which investigated the relationship between cortical excitability and visual oscillatory responses. As with the two previous studies, demonstrating that concurrent tDCS/MEG of the visual system is a feasible tool for investigating visual neuronal oscillations, Marshall et al. report that tDCS over the visual cortex did not change the amplitude of visual stimulus induced oscillatory responses. Publishing negative results from tES studies (e.g. Hanley et al $2015 b$ ) is essential to ensure an unbiased dataset can be used for meta-analyses, which have to date come under some criticism when used in the tES field (Horvath et al., 2015).

Veccio el al. studied the effects of tDCS on the functional coupling of the motor cortical network as measured by electroencephalography (EEG). As well as finding that the assessment of functional coupling of EEG rhythms might enhance understanding of tDCSinduced effects on cortical connectivity, they report that cortical connectivity was more effectively influenced by anodal stimulation compared to cathodal stimulation.

Toni Cunniliera and colleagues performed a tDCS-event-related-potential (ERP) coregistration study to investigate the role of the right inferior frontal cortex (rIFC) in response inhibition. They found that an online tDCS-ERP approach is achievable, but that a tDCS bilateral montage may not be the most efficient one for modulating the rIFC.

In another tDCS-EEG study, Randolph Helfrich and colleagues investigated the functional role of cross-frequency coupling, focusing on alpha and gamma-band oscillations for visual processing. They found that entrainment of the low-frequency component increased phaseamplitude-coupling (PAC), where gamma power became preferentially locked to the 'trough' or 'down-phase' of the alpha oscillation, while gamma-band entrainment enhanced amplitude-envelope correlations (AECs) and reduced alpha power. 
The diversity and complexity of possible mechanisms underlying tES effects is well exemplified by Dennis Schutter, who provides a systematic review on cutaneous retinal activation and neural entrainment in transcranial alternating current stimulation (tACS). Schutter argues that peripheral photic stimulation can at least in part explain the central effects that are attributed to classical tACS.

Witkowski et al. show that classical tACS stimulation protocols can lead to signal artifacts that cannot be removed by linear constraint magnetic variance (LCMV) beamformers. To solve this problem, the authors suggest to use amplitude modulation of a high-frequency stimulator signal oscillating at a frequency outside the physiological range, e.g. $220 \mathrm{~Hz}$. Using this technique, they show millimeter precise cortical mapping of entrained brain oscillations. In an intriguing study, Noury et al. show that physiological processes, such as heart beat and respiration, non-linearly affect electrophysiological recordings during classical tES protocols that could be mistaken as neural entrainment. Both studies underline the necessity to refine current analysis approaches and stimulation protocols in order to improve their interpretability.

Ivan Alekseichuk and colleagues performed transcranial electrical stimulation of the occipital cortex during visual perception and reports that such stimulation approach modifies the magnitude of blood oxygen level dependent (BOLD) activity. Alekseichuk et al. stresses that tES during visual perception can affect neuronal metabolism, which can be detected with functional magnetic resonance imaging ( $\mathrm{fMRI}$ ).

Johannes Vosskuhl and colleagues conducted a concurrent tACS-fMRI study investigating tACS effects in the alpha range on the BOLD signal. Whereas a direct effect of tACS on resting state BOLD signal levels could not be shown, Vosskuhl et al. found BOLD deactivation in an area where the BOLD signal was shown to correlate negatively with alpha amplitude. They conclude that tACS-related changes in BOLD activity may occur only as a modulation of an existing BOLD response.

Rachel Holland and colleagues studied the modulation of frontal effective connectivity during speech and identified the left inferior frontal cortex (IFC) as a 'top-down' hub and driver for speech change. They found that during anodal tDCS targeting the left frontal cortex individual variability in the feedforward connection strength from the inferior frontal sulcus (IFS) to ventral premotor (VPM) positively correlated with the degree of facilitation in naming behaviour.

Ehlis et al. used near infrared spectroscopy (NIRS) to demonstrate task-dependent and polarity-specific effects of prefrontal tDCS on cortical activation during word fluency. They found that neither anodal nor cathodal tDCS modulated verbal fluency task (VFT) performance. However, preconditioning with anodal tDCS increased brain activity during the VFT, whereas a trend for decreased activation was found after cathodal tDCS, a difference that was not found with simple speech production. Ehlis et al. underline that these findings support the notion of a tDCS-polarity-specific malleability of neuronal network activity associated with speech production. 
The papers above demonstrate that both the behavioural and imaging-based measurements of tES effects are very variable, suggesting that we have yet to fully appreciate the size of the parameter space of factors which ultimately underlie the success or failure of a given experiment. One metric which may be amenable to studies is variability in anatomy across participants. Robert Lindenberg investigated the neural correlates of unihemispheric and bihemispheric tDCS applied over the motor cortex in healthy young adults, and found stimulation effects to be highly variable across the study group. Lindenberg et al. suggest that dual tES exerts its effects via transcallosal motor tracts that could be assessed by diffusion tensor imaging (DTI). Thus, DTI could be used as a surrogate marker of tDCS response.

The availability of precise and valid head models is an important pre-requisite to optimize stimulation studies. Yu Huang and his colleagues developed a precise standardized volume conductor model for electroencephalographic (EEG) source localization and tES targeting, which they introduce as the ICBM (International Consortium for Brain Mapping)-NY or "The New York Head". Huang et al. show that this model outperforms finite element models (FEM) of mismatched individual anatomies as well as the boundary element models (BEM) of the ICBM anatomy.

Sven Wagner and colleagues investigated the relationship between tES and the EEG forward problem. Using Helmholtz reciprocity, a direct link between the EEG forward problem and tCS is presented. It is then shown that numerical accuracy for tCS simulations can be significantly improved using geometry-adapted hexahedral meshes in conjunction with an isoparametric finite element method (FEM) approach.

Finally, Tim Kunze and colleagues describes a large-scale brain network modelling study that indicates resting state functional connectivity changes during tES. This model indicates that synchronization is a key mechanism underlying transcranial direct current stimulation (tDCS) effects on changes in the spatiotemporal pattern formation. This corroborates previous work suggesting that NIBS is able to bias brain dynamics by affecting the competitive interplay of functional sub-networks.

\section{Conclusion}

The important findings reported in this Special Issue document impressive methodological advancements in combining tES and neuroimaging. At the same time, they also illustrate convincingly the many caveats and challenges associated with such studies, e.g. dealing with artifact cancellation or modeling between-participant anatomical variability. At present, combined $\mathrm{tES}$ and neuroimaging studies, represent only a small fraction of NIBS-related publications. With tES currently being subjected to much scrutiny from both within and outside the field, there is a need for multiple avenues to investigate the techniques underlying mechanisms of action. Animal studies are crucial, as are simulations, but the end goal for many groups is the combination of in-vivo stimulation and imaging to allow behaviour and neurobiology to be indexed in awake, behaving humans. In future, we hope that advancements in innovative neurotechnologies allowing for combinations of tES and real-time neuroimaging will further boost this impact. We sincerely hope that this Special Issue will contribute towards this end, and mark a starting point for fruitful discussions and the generation of new, novel avenues of research. 
Surjo R. Soekadar, Jim Don Herring and David J. McGonigle

Guest Editors

\section{References}

Alekseichuk I, Diers K, Paulus W, Antal A. Transcranial electrical stimulation of the occipital cortex during visual perception modifies the magnitude of BOLD activity: A combined tES-fMRI approach. Neuroimage. 2015 Nov 23. pii: S1053-8119(15)01056-3. doi: 10.1016/j.neuroimage.2015.11.034. [Epub ahead of print]

Bergmann TO, Karabanov A, Hartwigsen G, Thielscher A, Siebner HR. Combining non-invasive transcranial brain stimulation with neuroimaging and electrophysiology: Current approaches and future perspectives. Neuroimage. 2016 Feb 13. pii: S10538119(16)00119-1. doi: 10.1016/j.neuroimage.2016.02.012. [Epub ahead of print]

Cunillera T, Brignani D, Cucurell D, Fuentemilla L, Miniussi C.The right inferior frontal cortex in response inhibition: A tDCS-ERP co-registration study. Neuroimage. 2015 Nov 24. pii: S1053-8119(15)01066-6. doi: 10.1016/j.neuroimage.2015.11.044. [Epub ahead of print]

Ehlis AC, Haeussinger FB, Gastel A, Fallgatter AJ, Plewnia C. Task-dependent and polarityspecific effects of prefrontal transcranial direct current stimulation on cortical activation during word fluency. Neuroimage. 2015 Dec 31. pii: S1053-8119(15)01160-X. doi: 10.1016/j.neuroimage.2015.12.047. [Epub ahead of print]

Fitzgerald, F. (1941) The Last Tycoon. NY: Charles Scribner's Sons.

Garcia-Cossio E, Witkowski M, Robinson SE, Cohen LG, Birbaumer N, Soekadar SR. Simultaneous transcranial direct current stimulation (tDCS) and whole-head magnetoencephalography (MEG): assessing the impact of tDCS on slow cortical magnetic fields. Neuroimage. 2015 Oct 9. pii: S1053-8119(15)00891-5. doi: 10.1016/j.neuroimage.2015.09.068. [Epub ahead of print]

Hanley, C. J., Tommerdahl, M., \& McGonigle, D. J. (2015a). Stimulating somatosensory psychophysics: a double-blind, sham-controlled study of the neurobiological mechanisms of tDCS. Frontiers in Cellular Neuroscience, 9. http://doi.org/10.3389/fncel.2015.00400

Hanley CJ, Singh KD, McGonigle DJ. Transcranial modulation of brain oscillatory responses: A concurrent tDCS-MEG investigation. Neuroimage. 2015b Dec 17. pii: S10538119(15)01134-9. doi: 10.1016/j.neuroimage.2015.12.021. [Epub ahead of print]

Helfrich RF, Herrmann CS, Engel AK, Schneider TR. Different coupling modes mediate cortical cross-frequency interactions. Neuroimage. 2015 Nov 23. pii: S1053-8119(15)01057-5. doi: 10.1016/j.neuroimage.2015.11.035. [Epub ahead of print]

Holland R, Leff AP, Penny WD, Rothwell JC, Crinion J. Modulation of frontal effective connectivity during speech. Neuroimage. 2016 Jan 26. pii: S1053-8119(16)00052-5. doi: 10.1016/j.neuroimage.2016.01.037. [Epub ahead of print]

Horvath, J. C., Forte, J. D., \& Carter, O. (2015). Evidence that transcranial direct current stimulation (tDCS) generates little-to-no reliable neurophysiologic effect beyond MEP amplitude modulatio... - PubMed - NCBI. Neuropsychologia, 66, 213-236. http://doi.org/10.1016/j.neuropsychologia.2014.11.021

Huang Y, Parra LC, Haufe S. The New York Head-A precise standardized volume conductor model for EEG source localization and tES targeting. Neuroimage. 2015 Dec 17. pii: 
S1053-8119(15)01132-5. doi: 10.1016/j.neuroimage.2015.12.019. [Epub ahead of print] Kunze T, Hunold A, Haueisen J, Jirsa V, Spiegler A. Transcranial direct current stimulation changes resting state functional connectivity: A large-scale brain network modeling study. Neuroimage. 2016 Feb 13. pii: S1053-8119(16)00122-1. doi: 10.1016/j.neuroimage.2016.02.015. [Epub ahead of print]

Liew SL, Santarnecchi E, Buch ER, Cohen LG. Non-invasive brain stimulation in neurorehabilitation: local and distant effects for motor recovery. Front Hum Neurosci. 2014 Jun 27;8:378. doi: 10.3389/fnhum.2014.00378. eCollection 2014. Review.

Lindenberg R, Sieg MM, Meinzer M, Nachtigall L, Flöel A. Neural correlates of unihemispheric and bihemispheric motor cortex stimulation in healthy young adults. Neuroimage. 2016 Feb 3. pii: S1053-8119(16)00072-0. doi: 10.1016/j.neuroimage.2016.01.057. [Epub ahead of print]

Marshall TR, Esterer S, Herring JD, Bergmann TO, Jensen O. On the relationship between cortical excitability and visual oscillatory responses - A concurrent tDCS-MEG study. Neuroimage. 2015 Oct 9. pii: S1053-8119(15)00892-7. doi: 10.1016/j.neuroimage.2015.09.069. [Epub ahead of print]

Noury N, Hipp JF, Siegel M. Physiological processes non-linearly affect electrophysiological recordings during transcranial electric stimulation. Neuroimage. 2016 Apr 1. pii: S10538119(16)30009-X. doi: 10.1016/j.neuroimage.2016.03.065. [Epub ahead of print]

Paus, T. (1999). Imaging the brain before, during, and after transcranial magnetic stimulation. Neuropsychologia, 37(2), 219-224.

Schutter DJ. Cutaneous retinal activation and neural entrainment in transcranial alternating current stimulation: A systematic review. Neuroimage. 2015 Oct 8. pii: S10538119(15)00890-3. doi: 10.1016/j.neuroimage.2015.09.067. [Epub ahead of print]

Vecchio F, Pellicciari MC, Miraglia F, Brignani D, Miniussi C, Rossini PM. Effects of transcranial direct current stimulation on the functional coupling of the sensorimotor cortical network. Neuroimage. 2016 Jan 28. pii: S1053-8119(16)00066-5. doi: 10.1016/j.neuroimage.2016.01.051.

Vosskuhl J, Huster RJ, Herrmann CS. BOLD signal effects of transcranial alternating current stimulation (tACS) in the alpha range: A concurrent tACS-fMRI study. Neuroimage. 2015 Oct 10. pii: S1053-8119(15)00897-6. doi: 10.1016/j.neuroimage.2015.10.003. [Epub ahead of print]

Witkowski M, Garcia-Cossio E, Chander BS, Braun C, Birbaumer N, Robinson SE, Soekadar SR. Mapping entrained brain oscillations during transcranial alternating current stimulation (tACS). Neuroimage. 2015 Oct 17. pii: S1053-8119(15)00934-9. doi: 10.1016/j.neuroimage.2015.10.024. [Epub ahead of print] 